BULL. AUSTRAL. MATH. SOC.

$43 A 15$

VOL. $23(1981), 215-225$.

$(43 A 30,43 A 77)$

\title{
FACTORISATION OF LIPSCHITZ FUNCTIONS ON ZERO DIMENSIONAL GROUPS
}

\author{
WALTER R. BLOOM
}

Let $G$ denote a locally compact metrisable zero dimensional group with left translation invariant metric $d$. The Lipschitz spaces are defined by

$$
\operatorname{Lip}(\alpha ; r)=\left\{f \in L^{r}(G):\left\|_{a} f-f\right\|_{r}=O\left(d(a, 0)^{\alpha}\right), a \rightarrow 0\right\},
$$

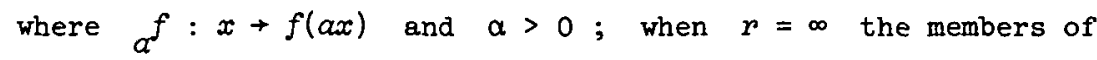
$\operatorname{Lip}(\alpha ; r)$ are taken to be continuous. For a suitable choice of metric it is shown that $\operatorname{Lip}(\alpha ; q) \subset L_{*}^{p} * L^{q}(G)$, where $1 \leq p \leq 2, \alpha>q^{-1}, p, q$ are conjugate indices and $L_{*}^{p}(G)=\left\{f: f^{*} \in L^{p}(G),\left(f^{*}(x)=f\left(x^{-1}\right)\right)\right\}$. It is also shown that for $G$ infinite the range of values of $\alpha$ cannot be extended.

The problem of factorising Lipschitz functions defined on real Euclidean space or the circle group has been considered by Hahn [4], Lohoue [7] and Uno [8]. Subsequently Uno [9] has proved that for compact metrisable zero dimensional abelian groups satisfying a certain boundedness condition (the so-called bounded Vilenkin groups) $\operatorname{Lip}(\alpha ; q) \subset L^{p} * L^{q}(G)$, where $1 \leq p \leq 2, \alpha>q^{-1}$ and $p, q$ are conjugate indices, that is,

Received 9 October 1980. This paper was prepared during a visit to the University of Tiubingen. The author would like to thank the Institute of Mathematics for its hospitality. 
$p^{-1}+q^{-1}=1$ (with the usual convention if $p=1$ ). The proof that Uno gives makes use of an ordering on the dual group, first introduced by Vilenkin [10].

We show that this result holds for any locally compact metrisable zero dimensional group $G$, the proof using only some simple properties of a certain bounded approximate unit for $L^{1}(G)$ (see Lemma 1 below).

Throughout $G$ will denote a locally compact metrisable zero dimensional group with right Haar measure $\lambda$. We take a neighbourhood basis $\left(V_{n}\right)$ at the identity consisting of a strictly decreasing sequence of compact open subgroups of $G$ (for the existence of such a basis see $[5,(7.7)]$; when $G$ is compact the $V_{n}$ are taken to be normal), $\left(B_{n}\right)$ to be any strictly decreasing sequence of positive numbers tending to zero, and $d$ defined on $G \times G$ by

$$
d(x, y)=\left\{\begin{array}{ll}
\beta_{n+1}, & y^{-1} x \in V_{n} \backslash V_{n+1} \\
\beta_{1}, & y^{-1} x \leqslant V_{1}, \\
0, & x=y
\end{array},\right.
$$

(see [11, section 2]). It is easily verified that $d$ is a left translation invariant metric on $G$ compatible with the given topology. We follow [11] and put $B_{n}=\lambda\left(V_{n}\right)$. This choice of metric agrees with that usually taken when $G$ is a product of finite cyclic groups, and includes that considered by Uno [9]. The Lipschitz spaces Lip $(\alpha ; r)$ will be defined as in the abstract with respect to the above metric. It should be noted that our choice of the strictly decreasing sequence $\left(v_{n}\right)$ is arbitrary .

We define $k_{n}=\lambda\left(V_{n}\right)^{-1} \xi_{n}$, where $\xi_{n}$ denotes the characteristic function of the set $V_{n}$. Clearly $k_{n} \geq 0$ and $\int_{G} k_{n} d \lambda=1$. Furthermore, by $[5,(20.15)],\left(k_{n}\right)$ is a bounded approximate unit for $L^{1}(G)$. We require two lemmas . 
LEMMA 1.

$$
\left(k_{m+1}-k_{m}\right) *\left(k_{n+1}-k_{n}\right)= \begin{cases}0, & m \neq n, \\ k_{n+1}-k_{n}, & m=n .\end{cases}
$$

Proof. First note that

$$
k_{m} * k_{n}(x)=\left(\lambda\left(v_{m}\right) \lambda\left(V_{n}\right)\right)^{-1} \lambda\left(v_{m} x \cap v_{n}\right) .
$$

Now suppose that $m \geq n$. For $x \in V_{n}, V_{m} x \cap V_{n}=V_{m} x$ and $k_{m} * k_{n}(x)=\lambda\left(V_{n}\right)^{-1}$. For $x \notin V_{n}, V_{m} x$ and $V_{n}$ are disjoint, and $k_{m} * k_{n}(x)=0$. Hence $k_{m} * k_{n}=k_{n}$ for $m \geq n$, from which the lemma follows.

LEMMA 2. Let $f \in \operatorname{Lip}(\alpha ; r)$. Then

$$
\left\|k_{n+1} \star f-k_{n} * f\right\|_{r} \leq 2 K \lambda\left(V_{n+1}\right)^{\alpha},
$$

where $K$ depends only on $f$.

Proof. For each $n$,

$$
k_{n} * f-f=\int_{G}(y-f) k_{n}\left(y^{-?]}\right) d \lambda(y)
$$

and, using Minkowski's inequality for integrals, for all $n$ suitably large,

$$
\begin{aligned}
\left\|k_{n} * f-f\right\|_{r} & \leq \int_{V_{n}}\left\|_{y} f-f\right\|_{r} k_{n}\left(y^{-1}\right) d \lambda(y) \\
& \leq K \sup \left\{d(y, 0)^{\alpha}: y \in V_{n}\right\} \\
& =K \lambda\left(V_{n+1}\right)^{\alpha} .
\end{aligned}
$$

We can assume that this inequality holds for all $n \geq 1$, and hence

$$
\left\|k_{n+1} * f-k_{n} * f\right\|_{r} \leq K\left(\lambda\left(v_{n+2}\right)^{\alpha}+\lambda\left(V_{n+1}\right)^{\alpha}\right) \leq 2 K \lambda\left(V_{n+1}\right)^{\alpha} \text {. }
$$

We now have our main result.

THEOREM 1. Let $1 \leq p \leq 2$. There exists $g \in L^{p}(G) \cap L_{*}^{p}(G)$ such 
that for all $f \in \operatorname{Lip}(\alpha ; q)$ with $\alpha>q^{-1}$, there exists $h \in L^{q}(G)$ satisfying $f=g * h$.

Proof. Choose $B \in\left(q^{-1}, \alpha\right)$ and put

$$
g=k_{1}+\sum_{n=1}^{\infty} \lambda\left(v_{n+1}\right)^{\beta}\left(k_{n+1}-k_{n}\right) \text {. }
$$

Clearly $g \in L^{p}(G) \cap L_{*}^{p}(G)$ as $k_{n}^{*}=k_{n}$ and

$$
\begin{aligned}
\|g\|_{p} & \leq\left\|k_{1}\right\|_{p}+\sum_{n=1}^{\infty} \lambda\left(V_{n+1}\right)^{\beta}\left(\left\|k_{n+1}\right\|_{p}+\left\|k_{n}\right\|_{p}\right) \\
& \leq \lambda\left(v_{1}\right)^{p^{-1}-1}+2 \sum_{n=1}^{\infty} \lambda\left(v_{n+1}\right)^{\beta+p^{-1}-1}<\infty,
\end{aligned}
$$

the last inequality following since $B+p^{-1}-1=\beta-q^{-1}>0$ and $\lambda\left(V_{n+1}\right) \leq 2^{-n} \lambda\left(v_{1}\right)$ (recall that $V_{n+1}$ is a proper subgroup of $v_{n}$ for each $n$ ). Now

$$
h=k_{1} * f+\sum_{n=1}^{\infty} \lambda\left(V_{n+1}\right)^{-B}\left(k_{n+1}-k_{n}\right) * f
$$

satisfies the conditions of the theorem. Indeed we have, from Lemma 1 ,

$$
g * h=k_{1} * f+\sum_{n=1}^{\infty}\left(k_{n+1}-k_{n}\right) * f=f \text {, }
$$

the second equality following from the property that $\left(k_{n}\right)$ is a bounded approximate unit for $L^{1}(G)$ (see the proof of Lemma 2, for example). Also Lemma 2 shows that

$$
\begin{aligned}
\|h\|_{q} & \leq\left\|k_{1} * f\right\|_{q}+\sum_{n=1}^{\infty} \lambda\left(v_{n+1}\right)^{-\beta}\left\|\left(k_{n+1}-k_{n}\right) * f\right\|_{q} \\
& \leq\left\|k_{1} * f\right\|_{q}+2 K \sum_{n=1}^{\infty} \lambda\left(v_{n+1}\right)^{\alpha-\beta}<\infty,
\end{aligned}
$$

so that $h \in L^{q}(G)$.

For infinite groups the range of values of $\alpha$ in Theorem 1 cannot be 
extended. This we show using some of the properties of random Fourier series, but first we require a preliminary result, which is of interest in its own right. We introduce some notation.

Let $G$ denote a compact group with dual object $\Sigma$, the set of equivalence classes of continuous irreducible unitary representations of $G$. For each $\sigma \in \Sigma$ fix a representative $U^{(\sigma)}$ and let $H_{\sigma}$ be the Hilbert space in which $U^{(\sigma)}$ acts. The (finite) dimension of $H_{\sigma}$ is denoted by $d_{\sigma}$. The Fourier series of $f \in L^{1}(G)$ is given by

$$
\sum_{\sigma \in \Sigma} d_{\sigma} \operatorname{tr}\left[\hat{f}(\sigma) U^{(\sigma)}(x)\right]
$$

where $\operatorname{tr}$ denotes the usual trace function and $\hat{f}(\sigma)$, the Fourier transform of $f$ at $\sigma$, is given by

$$
\hat{f}(\sigma)=\int_{G} f(x) U^{(\sigma)}\left(x^{-1}\right) d \lambda(x) \text {. }
$$

Write $E(\Sigma)=\prod_{\sigma \in \Sigma} B\left(H_{\sigma}\right)$, where $B\left(H_{\sigma}\right)$ denotes the space of linear operators on $H_{\sigma}$ and, for each $E=\left(E_{\sigma}\right) \in E(\Sigma)$, define the norm $\|E\|_{p}$ as in $[6,(28.34)]$,

$$
\|E\|_{p}=\left(\sum_{\sigma \in \Sigma} d_{\sigma}\left\|E_{\sigma}\right\|_{\varphi_{p}}^{p}\right)^{1 / p}
$$

where \|\|$_{\varphi_{p}}$ are the von Neumann norms of $[6,(D .37),(D .36)(e)]$. We put

$$
E_{p}(\Sigma)=\left\{E \in E(\Sigma):\|E\|_{p}<\infty\right\} \text {. }
$$

Note that by the Peter-Weyl theorem, the Fourier transformation $f \rightarrow \hat{f}$ is an isomorphism of the Hilbert spaces $L^{2}(G)$ and $E_{2}(\Sigma)$.

THEOREM 2. Let $G$ be compact and $1 \leq p \leq 2$. If $f \in \operatorname{Lip}(\alpha ; q)$ with $\alpha>q^{-1}$ then $\hat{f} \in E_{r}(\Sigma)$, where $r=2 p(3 p-2)^{-1}$.

Proof. From Theorem 1 we have 


$$
\operatorname{Lip}(\alpha ; q) \subset L^{p} * L^{q}(G) \subset L^{p} * L^{2}(G)
$$

and, using $[6,(28.36),(28.43)$ and $(31.25)]$, we have that $\hat{f} \in E_{q}(\Sigma) E_{2}(\Sigma)$. The result now follows by appealing to $[6,(28.33)]$.

Theorem 2, which is an extension of a classical theorem of Bernstein on the absolute convergence of Fourier series, has been obtained previously for $G$ abelian (see [2, Theorem 2]), where it was also shown ([2, Theorem 4]) that the range of values of $\alpha$ cannot be extended. For $G$ not necessarily abelian Benke ([1, Corollary, p. 323]) has a version of our Theorem 2, but only for $p=2$ and for $G$ satisfying a certain boundedness condition. Benke also shows that his result is sharp (see [1, Theorem 3]).

We can now prove that the results of Theorem 1 are sharp.

THEOREM 3. Suppose $G$ is infinite and let $1<p \leq 2$. There exists $f \in \operatorname{Lip}\left(q^{-1} ; q\right)$ with $f \neq I_{*}^{p} * L^{q}(G)$.

Theorem 3 would also hold for $p=1$, provided $\operatorname{Lip}(0 ; \infty)$ is defined to be $L^{\infty}(G)$. In this case the result would state that there existed bounded functions that are not continuous.

Our proof of the theorem is divided into two cases:

(i) $G$ noncompact

Choose a sequence $\left\{x_{n}\right\}$ with $x_{1}=1$ and satisfying $V_{1} x_{m} \cap V_{1} x_{n}=\emptyset$ for $m \neq n$, and write $f=\sum_{n=1}^{\infty} \eta_{n}$, where $\eta_{n}$ denotes the characteristic function of $V_{n} x_{n}$. We show that $f \in \operatorname{Lip}\left(q^{-1} ; q\right)$. First note that $f \in L^{r}(G)$ for all $r \geq 1$, since

$$
\|f\|_{r}^{p}=\sum_{n=1}^{\infty} \lambda\left(V_{n} x_{n}\right)=\sum_{n=1}^{\infty} \lambda\left(V_{n}\right) \leq \lambda\left(V_{1}\right) \sum_{n=1}^{\infty} 2^{1-n}=2 \lambda\left(V_{1}\right) .
$$

Furthermore, for $a \in V_{k} \backslash V_{k+1}$ we see that $a^{n_{n}}=\eta_{n}$ if $n \leq k$ and $\left\|_{a} n_{n}\right\|_{q}=\left\|n_{n}\right\|_{q}$ for all $n$; for the latter equality just use the property that the Haar measure of the compact (hence unimodular) group $V_{1}$ is just 
the restriction of $\lambda$ to $V_{1}$, suitably normalised. It follows that for such $a$,

$$
\left\|_{\alpha} f-f\right\|_{q} \leq 2 \sum_{n=k+1}^{\infty}\left\|n_{n}\right\|_{q}=2 \sum_{n=k+1}^{\infty} \lambda\left(v_{n}\right)^{q^{-1}}=K d(a, 0)^{q^{-1}}
$$

for some constant $K$, and thus $f \in \operatorname{Lip}\left(q^{-1} ; q\right)$. However it is clear that $f \notin C_{0}(G)$ (the space of continuous functions on $G$ vanishing at infinity) and hence $f \neq L_{*}^{p} * L^{q}(G)$ (see $\left.[5,(20.32)(\mathrm{e})]\right)$.

(ii) $G$ compact

In view of the proof of Theorem 2 we need only exhibit $f \in \operatorname{Lip}\left(q^{-1} ; q\right)$ having $\hat{f} \vDash E_{r}(\Sigma)$, where $r=2 p(3 p-2)^{-1}$. We consider $k_{n}=\lambda\left(v_{n}\right)^{-1} \xi_{n}$, introduced in the introduction. It is clear that the restriction $\omega$ of $k_{n} d \lambda$ to $V_{n}$ is just the normalised Haar measure on $V_{n}$ and, by $[6,(28.72)(\mathrm{g})]$ (note that since $G$ is compact we have that $V_{n}$ is normal),

$$
\{\sigma \in \Sigma: \hat{\omega}(\sigma) \neq 0\}=\{\sigma \in \Sigma: \hat{\omega}(\sigma)=I\}=A_{n},
$$

where $A_{n}=\left\{\sigma \in \Sigma: U^{(\sigma)}(x)=I\right.$ for all $\left.x \in V_{n}\right\}$ is the annihilator of $V_{n}$ in $\Sigma$. In particular the Fourier series of $k_{n}$ is given by

$$
\sum_{\sigma \in A_{n}} d_{\sigma} \operatorname{tr}\left[U^{(\sigma)}(x)\right]
$$

Now by [3, Theorem 4], $W \in E(\Sigma)$ with each $W_{\sigma}$ unitary can be chosen so that

$$
\sum_{\sigma \in A_{n+1} A_{n}} d_{\sigma} \operatorname{tr}\left[W_{\sigma} U^{(\sigma)}(x)\right]
$$

is the Fourier series of a function $z_{n} \in L^{q}(G)$, where $\left\|z_{n}\right\|_{q} \leq K(q)\left\|z_{n}\right\|_{2}$ and $K(q)$ is a constant depending only on $q$. Define 


$$
f=\sum_{n=1}^{\infty} \lambda\left(v_{n+1}\right)^{q^{-1}+\frac{3}{2}} \tau_{n}
$$

Using the equalities

$$
\left\|\tau_{n}\right\|_{2}=\left\|k_{n+1}-k_{n}\right\|_{2}=\left(\lambda\left(v_{n+1}\right)^{-1}-\lambda\left(v_{n}\right)^{-1}\right)^{\frac{3}{2}}
$$

we have

$$
\|f\|_{q} \leq \sum_{n=1}^{\infty} \lambda\left(v_{n+1}\right)^{q^{-1}+\frac{1}{2}} K(q)\left(\lambda\left(v_{n+1}\right)^{-1}-\lambda\left(v_{n}\right)^{-1}\right)^{\frac{1}{2}}<\infty,
$$

so that $f \in L^{q}(G)$. Furthermore, for $a \in V_{k} \backslash V_{k+1}$ and $n \leq k-1$ we see that

$$
a^{2} z_{n}-z_{n}=a^{k}{ }_{n+1} * z_{n}-k_{n+1} * z_{n}=\left(a_{n+1}-k_{n+1}\right) * z_{n}=0
$$

and, for some constant $C(q)$,

$$
\begin{aligned}
\left\|_{a} f-f\right\|_{q} & \leq \sum_{n=k}^{\infty} \lambda\left(v_{n+1}\right)^{q^{-1}+\frac{1}{2}} 2\left\|z_{n}\right\|_{q} \\
& \leq 2 K(q) \sum_{n=k}^{\infty} \lambda\left(v_{n+1}\right)^{q^{-1}+\frac{1}{2}}\left(\lambda\left(v_{n+1}\right)^{-1}-\lambda\left(v_{n}\right)^{-1}\right)^{\frac{1}{2}} \\
& \leq C(q) \lambda\left(v_{k+1}\right)^{q^{-1}}=C(q) d(a, 0)^{q^{-1}}
\end{aligned}
$$

which shows that $f \in \operatorname{Lip}\left(q^{-1} ; q\right)$.

Now, using the property that the spectra of the $z_{n}$ are pairwise disjoint, 


$$
\begin{aligned}
\|\hat{f}\|_{r}^{r} & =\sum_{\sigma \in \Sigma} a_{\sigma}\|\hat{f}(\sigma)\|_{\varphi_{r}}^{r} \\
& \geq \sum_{n=1}^{\infty} \sum_{\sigma \in A_{n+1} V_{n}} d_{\sigma} \lambda\left(v_{n+1}\right) r\left(q^{-1}+\frac{3}{2}\right)\left\|\hat{\tau}_{n}(\sigma)\right\|_{\varphi_{r}}^{r} \\
& =\sum_{n=1}^{\infty} \lambda\left(V_{n+1}\right) \sum_{\sigma \in A_{n+1} V_{n}} d_{\sigma}\left\|W_{\sigma}\right\|_{\varphi_{r}}^{r} \\
& =\sum_{n=1}^{\infty} \lambda\left(V_{n+1}\right) \sum_{\sigma \in A_{n+1} V_{n}} d_{\sigma}^{2} .
\end{aligned}
$$

As $V_{n+1}$ is a proper subgroup of $V_{n}$ we have $\lambda\left(v_{n}\right)-\lambda\left(V_{n+1}\right) \geq \frac{1}{2} \lambda\left(v_{n+1}\right)$. It follows that

$$
\sum_{\sigma \in A_{n+1} A_{n}} d_{\sigma}^{2}=\left\|k_{n+1}-k_{n}\right\|_{2}^{2} \geq \frac{z}{2} \lambda\left(V_{n+1}\right)^{-1},
$$

and so we deduce that $\hat{f} \notin E_{p}(\Sigma)$. This completes the proof of Theorem 3 .

Theorem 1 admits the following generalisation. We suppose that $1 \leq p_{1} \leq \ldots \leq p_{m} \leq \infty$ are given with $p_{1}^{-1}+\ldots+p_{m}^{-1}=m-1$, where $m \geq 2$. If $q_{j}$ denotes the index conjugate to $p_{j}$ then each $B>p_{m}^{-1}$ can be written as $\beta=\beta_{1}+\ldots+\beta_{m-1}$ with $\beta_{j}>q_{j}^{-1}, 1 \leq j \leq m-1$, since $q_{1}^{-1}+\ldots+q_{m-1}^{-1}=p_{m}^{-1}$. For each $1 \leq j \leq m-1$ define

$$
g_{j}=k_{1}+\sum_{n=1}^{\infty} \lambda\left(v_{n+1}\right)^{\beta_{j}}\left(k_{n+1}-k_{n}\right) .
$$

Then, using the notation of the proof of Theorem 1, we have

$$
g=g_{1} * \ldots * g_{m-1},
$$

where $g_{j} \in L^{p}{ }^{j}(G)$. Hence we obtain

THEOREM 4. Let $p_{j}, 1 \leq j \leq m$, be as above. Then, for $a>p_{m}^{-1}$,

$$
\operatorname{Lip}\left(\alpha ; p_{m}\right) \subset L^{p_{1}}(G) \star \ldots * L^{p_{m}}(G)
$$


The above details follow that of [9, Section 6], where the result is given for bounded Vilenkin groups.

\section{References}

[1] George Benke, "Smoothness and absolute convergence of Fourier series in compact totally disconnected groups", J. Funct. Anal. 29 (1978), 319-327.

[2] Walter R. Bloom, "Absolute convergence of Fourier series on totally disconnected groups", submitted.

[3] Alessandro Figà-Talamanca and Daniel Rider, "A theorem of Littlewood and lacunary series for compact groups", Pacific J. Math. 16 (1966), 505-514.

[4] Liang-Shin Hahn, "On multipliers of p-integrable functions", Trans. Amer. Math. Soc. 128 (1967), 321-335.

[5] Edwin Hewitt and Kenneth A. Ross, Abstract harmonic analysis I. Structure of topological groups, integration theory, group representations (Die Grundlehren der mathematischen Wissenschaften, 115. Springer-Verlag, Berlin, Heidelberg, New York, 1963).

[6] Edwin Hewitt and Kenneth A. Ross, Abstract harmonic conalysis II. Structure and analysis for compact groups. Analysis on locally compact abelian groups (Die Grundlehren der mathematischen Wissenschaften, 152. Springer-Verlag, Berlin, Heidelberg, New York, 1970).

[7] Noël Lohoue, "Une condition d'appartenance à $A_{p}(T)$ ", C.R. Acad. Sci. Paris Sér. A 270 (1970), 736-738.

[8] Yoshikazu Uno, "Lipschitz functions and convolution", Proc. Japan Acad. 50 (1974), 785-788.

[9] Yoshikazu Uno, "Lipschitz functions and convolution on bounded Vilenkin groups", Sci. Rep. Kanazcawa Univ. 23 (1978), 1-6. 
[10] В.Я. Виленннн [N.Ja. Vilenkin], "Об одном нлассе полньх ортонормальньх снстем" [On a class of complete orthonormal systems], Izv. Akad. Nauk SSSR Ser. Mat. 11 (1947), 363-400; English Transl: Amer. Math. Soc. Trans 2 . (2) 28 (1963), 1-35.

[11] P.L. Walker, "Lipschitz classes on 0-dimensional groups", Proc. Combridge Philos. Soc. 63 (1967), 923-928.

School of Mathematical and Physical Sciences,

Murdoch University,

Murdoch,

Western Australia 6150. 